\title{
IDENTIFYING COMMON SOURCE DIGITAL CAMERA FROM IMAGE PAIRS
}

\author{
Miroslav Goljan, Mo Chen, Jessica Fridrich \\ Department of Electrical and Computer Engineering \\ Binghamton University, Binghamton, NY 13902-6000
}

\begin{abstract}
In this paper, we propose a method for verifying whether two digital images were obtained using the same digital camera. The method uses test statistics derived from a twochannel detector taking as input the noise residuals from both images. It is not assumed that the camera that took the images is available.
\end{abstract}

Index Terms - Forensics, camera identification, sensor, fingerprint, authentication

\section{INTRODUCTION}

Digital images are increasingly more often produced as silent witness in court and become a very crucial piece of evidence, e.g., in child pornography, movie piracy cases, or insurance claims. Therefore, the question of verifying their integrity and origin is rapidly increasing on importance.

In Source Classification, we wish to assign a given image to one of several broad classes, such as scan vs. digital camera, or Canon vs. Kodak, etc. Device Identification focuses on proving that a given image was obtained by a specific device that is available (prove that a given camera took a certain image or video). Finally, Device Linking is aiming to group objects according to their common source. For example, given a set of images, we would like to find out which images were obtained using the exact same camera. In a special case, we have two images and want to know if they came from the same camera. Note that it is not assumed that the camera or any other images from it are available. This is the task investigated in this paper.

One of the first methods for source classification of digital cameras was based on classifying features extracted from images [1] or estimated from color filter array (CFA) interpolation artifacts [2]. Another method for source classification [3] estimates the color interpolation kernel and then performs classification using machine learning. We note that source classification methods cannot be used for device identification or device linking as they only assign images to broad classes.

Device identification or linking require a "fingerprint" that is unique to a specific camera, such as defective pixels (hot or dead pixels) as originally proposed in [4]. The biggest limitation of using defective pixels as sensor fingerprint is that some sensors do not exhibit any detectable defects or have the defects corrected for. Moreover, some defective pixels may not be detectable in all images (e.g., a hot pixel with a blue filter in CFA will not be convincingly detectable in the sky). Recently, the photoresponse non-uniformity (PRNU) was proposed in $[5,6]$ as a sensor fingerprint, an equivalent of biometric for imaging sensors. The PRNU is inherent to all semiconductor imaging sensors, including CCD and CMOS architectures. It is relatively stable in time and can be reliably detected in virtually all images even after lossy compression or additional processing. The power of this approach is that the fingerprint signal is large (as large as the image dimension) and can be reliably detected using methods commonly used in spread spectrum watermark detection [7].

In this paper, we propose a method for device linking that uses the PRNU as a unique sensor fingerprint. In Section 2, we describe a simplified linearized model of imaging sensor output. The model is used in Section 3 to derive a detector for device linking. Experimental results are in Section 4. The paper is summarized in Section 5.

\section{LINEARIZED SENSOR OUTPUT MODEL}

We accept the following simplified model of the sensor output taken from $[6,8]$. Let $\mathbf{I}[i, j]$ be the signal in one color channel at pixel $[i, j], i=1, \ldots, m, j=1, \ldots, n$, where $m, n$ are image dimensions, generated by the sensor before demosaicking is applied. Denote by $\mathbf{Y}[i, j]$ the incident light intensity at the same pixel. Dropping the pixel indices for better readability, we have the following model of the sensor output

$$
\mathbf{I}=g^{\gamma} \cdot\left[(\mathbf{1}+\mathbf{K}) \mathbf{Y}+\boldsymbol{\Theta}_{n}\right]^{\gamma}+\boldsymbol{\Theta}_{q},
$$

where $g$ is the color channel gain, $\gamma$ is the gamma correction factor (typically, $\gamma \approx 1 / 2.2$ ), $\mathbf{K}$ is a zero-mean multiplicative factor responsible for PRNU, $\boldsymbol{\Theta}_{n}$ is a noise term that includes dark current, shot noise, read-out noise, and $\boldsymbol{\Theta}_{q}$ is the quantization noise. The gain factor $g$ adjusts the pixel intensity level according to the sensitivity of the pixel in the red, green, and blue spectral bands to obtain the correct white balance. We remind that all operations in (1) are element-wise. 
In our effort to develop a method applicable to a wide class of cameras, the model (1) does not incorporate specific postprocessing, such as color interpolation (demosaicking) or color correction. Since forensic methods based on this simplified model have been very successful in the past [6], we believe that neglecting the influence of postprocessing is justifiable.

Because the dominant term in the square bracket is the light intensity $\mathbf{Y}$, we can factor it out and apply Taylor expansion of $(1+x)^{\gamma}=1+\gamma x+O\left(x^{2}\right)$ to obtain

$$
\mathbf{I}=\mathbf{I}^{(0)}+\gamma \mathbf{I}^{(0)} \mathbf{K}+\boldsymbol{\Theta},
$$

where $\mathbf{I}^{(0)}=(g \mathbf{Y})^{\gamma}$ is the sensor output in the absence of noise and $\boldsymbol{\Theta}$ is a complex of independent random noise components.

We note that the signal of interest $\gamma \mathbf{I}^{(0)} \mathbf{K}$ is weak with a SNR typically around $-51 \mathrm{~dB}$, depending on the camera.

\section{DEVICE LINKING ALGORITHM}

We are studying the following problem: given two images $\mathbf{I}_{1}$ and $\mathbf{I}_{2}$, we desire to decide if they were taken with the same camera (that is unavailable). In the current version of the proposed method, we assume that no geometrical transformations besides cropping were applied to them (point-wise processing, such as filtering, lossy compression, or gamma correction, are allowed).

First, we perform host signal rejection to improve the SNR between $\gamma \mathbf{I}^{(0)} \mathbf{K}$ and observed data by subtracting from both sides of (2) an estimate $\hat{\mathbf{I}}^{(0)}=F(\mathbf{I})$ of $\mathbf{I}^{(0)}$ obtained using a denoising filter $F$

$$
\begin{aligned}
\mathbf{W} & =\mathbf{I}-\hat{\mathbf{I}}^{(0)}=\gamma \mathbf{I} \mathbf{K}+\mathbf{I}^{(0)}-\hat{\mathbf{I}}^{(0)}+\gamma\left(\mathbf{I}^{(0)}-\mathbf{I}\right) \mathbf{K}+\boldsymbol{\Theta} \\
& =\gamma \mathbf{I} \mathbf{K}+\mathbf{\Xi} .
\end{aligned}
$$

The term $\boldsymbol{\Xi}$ is a combination of $\boldsymbol{\Theta}$ with the additional distortion introduced by the denoising filter. Working with the noise residual $\mathbf{W}$ significantly improves the SNR for our signal of interest IK and thus improves the reliability of the image linking process. Additionally, we preprocess $\mathbf{W}$ using the row and column zero-meaning [7] to suppress some subtle artifacts due to color interpolation.

We use a wavelet based denoising filter that removes from images Gaussian noise with variance $\sigma_{F}^{2}$ (in this paper, we used $\sigma_{F}^{2}=3$ ). See [5] or [9] for more details about this filter.

For the null hypothesis, we include the weak PRNU terms coming from different cameras into the noise term and arrive at the following hypothesis testing problem:

$$
\begin{array}{ll}
\mathrm{H}_{0}: \mathbf{W}_{1}=\mathbf{\Xi}_{1}, & \mathbf{W}_{2}=\mathbf{\Xi}_{2} \\
\mathrm{H}_{1}: \mathbf{W}_{1}=\gamma \mathbf{I}_{1} \mathbf{K}+\mathbf{\Xi}_{1}, & \mathbf{W}_{2}=\gamma \mathbf{I}_{2} \mathbf{K}+\mathbf{\Xi}_{2},
\end{array}
$$

where $\mathbf{K}$ is an unknown signal. Here, we really should have written $\mathrm{H}_{0}: \mathbf{W}_{1}=\gamma \mathbf{I}_{1} \mathbf{K}+\mathbf{\Xi}_{1}, \mathbf{W}_{2}=\gamma \mathbf{I}_{1} \mathbf{K}{ }^{\prime}+\mathbf{\Xi}_{2}$, where $\mathbf{K}^{\prime}$ is a PRNU from some other camera. However, since the PRNU term is a weak signal and PRNUs from different cameras are independent, in (4) we included the PRNU terms into the noise terms.

The noise is modeled as a sequence of iid Gaussian variables with known variances $\sigma_{1}^{2}=\operatorname{var}\left(\mathbf{W}_{1}\right)$ and $\sigma_{2}^{2}=\operatorname{var}\left(\mathbf{W}_{2}\right)$. The optimal detector for this two-channel model has been derived by Holt [10]. In this paper, we present its slightly more general version when an unknown circular spatial shift $\boldsymbol{s}=(u, v)$ between the channels may exist (one of the images has been cropped). The optimal test statistics is a sum of three terms: two energy-like quantities and a cross-correlation term:

$$
T\left(\mathbf{I}_{1}, \mathbf{I}_{2}\right)=\max _{s}\left\{E_{1}(s)+E_{2}(s)+C(s)\right\}
$$

with

$$
\begin{aligned}
& E_{1}(s)=\sum_{i, j} \frac{\mathbf{I}_{1}^{2}[i, j] \mathbf{W}_{1}^{2}[i+u, j+v]}{\sigma_{1}^{2} \mathbf{I}_{1}^{2}[i, j]+\sigma_{1}^{4} \sigma_{2}^{-2} \mathbf{I}_{2}^{2}[i+u, j+v]}, \\
& E_{2}(s)=\sum_{i, j} \frac{\mathbf{I}_{2}^{2}[i+u, j+v] \mathbf{W}_{2}^{2}[i+u, j+v]}{\sigma_{2}^{2} \mathbf{I}_{2}^{2}[i+u, j+v]+\sigma_{2}^{4} \sigma_{1}^{-2} \mathbf{I}_{1}^{2}[i, j]}, \\
& C(s)=\sum_{i, j} \frac{\mathbf{I}_{1}[i, j] \mathbf{W}_{1}[i, j] \mathbf{I}_{2}[i+u, j+v] \mathbf{W}_{2}[i+u, j+v]}{\sigma_{2}^{2} \mathbf{I}_{1}^{2}[i, j]+\sigma_{1}^{2} \mathbf{I}_{2}^{2}[i+u, j+v]} .
\end{aligned}
$$

Unfortunately, the complexity of evaluating these three expressions is proportional to the square of the number of pixels because they cannot be evaluated using fast Fourier transform as a simple correlation can. For a 4 megapixel image, the required computing time on a state-of-the-art PC was of the order of days. As using the optimal detector would prevent us from running necessary extensive experiments, we decided to use a fast suboptimal detector instead. The energy terms $E_{1}$ and $E_{2}$ vary only little and slowly with $\boldsymbol{s}$. The maximum in (5) is due to the contribution of the cross-correlation term that exhibits a sharp peak when both images are from the same camera. Thus, we selected as an alternative test statistics the following NCC (normalized cross-correlation) easily calculable using FFT

$$
N C C(u, v)=\frac{\sum_{i, j}(\mathbf{X}[i, j]-\overline{\mathbf{X}})(\mathbf{Y}[i+u, j+v]-\overline{\mathbf{Y}})}{\sqrt{\sum_{i, j}(\mathbf{X}[i, j]-\overline{\mathbf{X}})^{2}} \sqrt{\sum_{i, j}(\mathbf{Y}[i, j]-\overline{\mathbf{Y}})^{2}}},
$$

where

$$
\mathbf{X}=\frac{\mathbf{I}_{1} \mathbf{W}_{1}}{\sqrt{\sigma_{2}^{2} \mathbf{I}_{1}^{2}+\sigma_{1}^{2} \mathbf{I}_{2}^{2}}}, \mathbf{Y}=\frac{\mathbf{I}_{2} \mathbf{W}_{2}}{\sqrt{\sigma_{2}^{2} \mathbf{I}_{1}^{2}+\sigma_{1}^{2} \mathbf{I}_{2}^{2}}}
$$

with bar denoting the sample mean.

A pronounced, sharp peak in NCC is indicative of the fact that both images were taken with the same camera (see Fig. 1a). There exist several different measures of peak sharpness [11]. One of them is the ratio between the primary peak to the secondary peak (PSR) defined as the largest value in the NCC excluding a central region around the primary peak. The size of this region is determined by observing when the NCC first drops to half of the primary peak. 
Based on a set of limited experimental comparisons, the suboptimal detector produces a peak in the NCC that is as sharp as the peak for the optimal statistics (when measured using PSR). In Fig. 1a,b, we show the optimal statistics and the suboptimal statistics for the range $-50 \leq u \leq 50,-50 \leq v$ $\leq 50$ for a pair of two aligned images produced by the same camera. Fig. 1c,d shows the same for a pair of images not coming from the same camera.

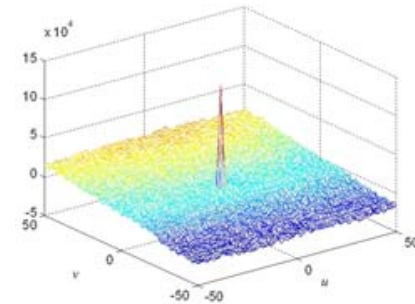

(a)

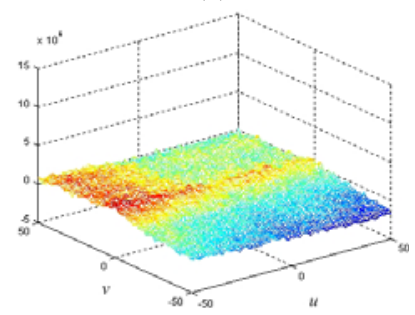

(c)

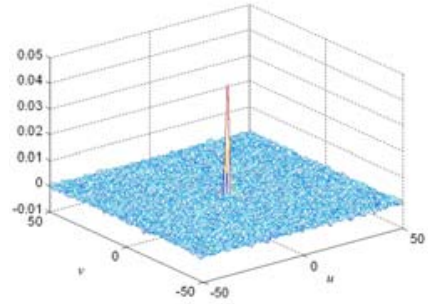

(b)

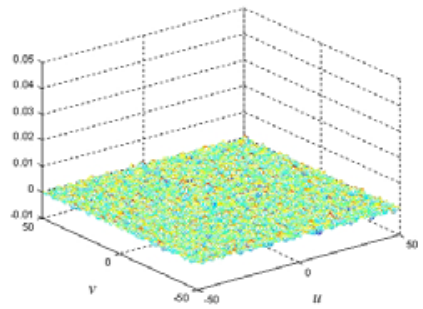

(d)
Figure 1: NCC for optimal (a) and suboptimal statistics (b) for a matched image pair (the top left and the top middle in Fig. 2, both are JPEG images from the same Canon G2 camera); (c) and (d) show the same for an unmatched pair (the top left and the top right in Fig. 2, one from the Canon G2 camera, and the other from Olympus C765-1 camera, both are JPEG images with quality 90).

In this paper, we use the PSR to evaluate the performance of the proposed method. An image pair is declared to come from the same camera if PSR $\geq T h$, where Th is a threshold selected to obtain a desired false positive rate (falsely identifying an image pair as coming from the same camera). From the Central Limit Theorem, the crosscorrelation values for non-matching images are well approximated using Gaussian distribution. The cumulative density function (cdf) of the PSR for $n$ samples taken from a Gaussian distribution with pdf $f(x)$ and $\operatorname{cdf} F(x)$ is

$$
c(z)=1-n z \int_{-\infty}^{\infty} f(x z) F^{n-1}(x) d x, z \geq 1 .
$$

Thus, setting the threshold to Th will produce the false alarm rate of

$$
P_{F A}=1-c(T h) \text {. }
$$

\section{EXPERIMENTS}

In our experiments, we have used images coming from 8 cameras from different manufacturers with a variety of sensors and resolutions. They included 6 CCD cameras Canon G2, Canon S40, Kodak DC290, Olympus C3030, Olympus C765 (two cameras of the exact brand), and two CMOS cameras - Sigma SD9 with the Foveon sensor and Canon XT Rebel.

Total of 10 images of various indoor and outdoor scenes in the raw format were taken with each camera. Then, for each camera, we ran the device linking algorithm for matching and non-matching image pairs. We tested all $10 \times 9 / 2=45$ matching pairs and 200 randomly chosen pairs where the first image was among the 10 images taken by the camera and the other image came from the remaining 7 cameras. For each test, we registered the PSR value. Some statistics (range and median) of the PSR values are displayed in Table 1. Figure 2 shows a sample of 9 images from the tested cameras.

To see how the reliability of the device linking algorithm deteriorates with lossy compression, we repeated the same experiment after all images were compressed using JPEG with quality factor 90 and 75 . The results are also shown in Table 1.

Regardless of the quality factor, the largest value of the PSR for an unmatched pair (among $3 \times 8 \times 200$ pairs) was 1.3 , while the smallest value for a matched pair (out of $3 \times 8 \times 45$ pairs) was 1.0 . Setting $T h=1.4$ would in our test produce zero false alarms (incorrectly classified non-matching pair) with the probability of false alarm (8) $P_{F A} \cong 5 \times 10^{-5}$. In Table 1 we report the percentage of correctly classified matching pairs with this theoretical false alarm rate. For example, 41 correctly classified cases out of 45 pairs of the raw Canon Rebel images result in $91.1 \%$ probability of correct detection of a matched pair (PDM).

The PDM is usually very high for raw images but deteriorates with a decreasing JPEG quality factor. Since the PRNU term IK is multiplicative, very dark images are more likely to be misclassified. The same is also true for highly textured images due to the limitation of the denoising filter, which fails to filter out the image content.

\section{SUMMARY}

We present a method that can be used to verify whether two digital images were obtained using the same digital camera. It is based on detecting a common component (the photoresponse non-uniformity previously proposed as a unique fingerprint for digital imaging sensors) in the noise residuals of both images. The current version of the method is applicable to JPEG compressed or cropped images and is expected to perform well for other point-wise processing. The method does not need the camera that took the images or any other auxiliary images. 


\section{ACKNOWLEDGEMENT}

The work on this paper was supported by AFOSR grant number FA9550-06-1-0046. The U.S. Government is authorized to reproduce and distribute reprints for Governmental purposes notwithstanding any copyright notation there on. The views and conclusions contained herein are those of the authors and should not be interpreted as necessarily representing the official policies, either expressed or implied, of Air Force Research Laboratory, or the U. S. Government.

\begin{tabular}{|c|c|c|c|c|c|c|c|c|c|c|c|c|c|c|c|c|c|}
\hline & & \multicolumn{3}{|c|}{ Matched Pairs } & \multicolumn{3}{|c|}{ Unmatched Pairs } & \multirow{2}{*}{ PDM } & & & \multicolumn{3}{|c|}{ Matched Pairs } & \multicolumn{3}{|c|}{ Unmatched Pairs } & \multirow{2}{*}{ PDM } \\
\hline & & $\min$ & med & $\max$ & $\min$ & med & $\max$ & & & & $\min$ & med & $\max$ & $\min$ & med & $\max$ & \\
\hline \multirow{3}{*}{$\begin{array}{l}\text { Canon } \\
\text { G2 }\end{array}$} & Raw & 7.4 & 11.6 & 24.3 & 1.00 & 1.03 & 1.19 & $100 \%$ & \multirow{3}{*}{$\begin{array}{c}\text { Olympus } \\
\text { C765-1 }\end{array}$} & Raw & 3.6 & 5.5 & 9.1 & 1.00 & 1.04 & 1.28 & $100 \%$ \\
\hline & Q90 & 4.1 & 6.6 & 16.5 & 1.00 & 1.03 & 1.20 & $100 \%$ & & Q90 & 1.8 & 3.6 & 4.8 & 1.00 & 1.03 & 1.25 & $100 \%$ \\
\hline & Q75 & 1.2 & 2.6 & 6.3 & 1.00 & 1.03 & 1.28 & $97.8 \%$ & & Q75 & 1.2 & 1.8 & 2.9 & 1.00 & 1.03 & 1.26 & $88.9 \%$ \\
\hline \multirow{3}{*}{$\begin{array}{l}\text { Canon } \\
\text { S40 }\end{array}$} & Raw & 8.8 & 12.6 & 23.2 & 1.00 & 1.03 & 1.30 & $100 \%$ & \multirow{3}{*}{$\begin{array}{c}\text { Olympus } \\
\text { C765-2 }\end{array}$} & Raw & 1.9 & 3.0 & 8.3 & 1.00 & 1.03 & 1.29 & $100 \%$ \\
\hline & Q90 & 5.3 & 8.4 & 14.3 & 1.00 & 1.03 & 1.30 & $100 \%$ & & Q90 & 1.1 & 1.9 & 4.6 & 1.00 & 1.03 & 1.24 & $86.7 \%$ \\
\hline & Q75 & 2.2 & 3.3 & 5.2 & 1.00 & 1.03 & 1.30 & $100 \%$ & & Q75 & 1.0 & 1.2 & 2.7 & 1.00 & 1.04 & 1.26 & $33.3 \%$ \\
\hline \multirow{3}{*}{$\begin{array}{c}\text { Canon XT } \\
\text { Rebel }\end{array}$} & Raw & 1.0 & 2.9 & 5.7 & 1.00 & 1.03 & 1.21 & $91.1 \%$ & \multirow{3}{*}{$\begin{array}{l}\text { Olympus } \\
\text { C3030 }\end{array}$} & SHQ & 8.4 & 15.0 & 28.1 & 1.00 & 1.04 & 1.26 & $100 \%$ \\
\hline & Q90 & 1.0 & 1.7 & 2.6 & 1.00 & 1.03 & 1.30 & $57.8 \%$ & & Q90 & 4.7 & 8.0 & 15.1 & 1.00 & 1.04 & 1.25 & $100 \%$ \\
\hline & Q75 & 1.0 & 1.1 & 1.6 & 1.00 & 1.04 & 1.27 & $4.4 \%$ & & Q75 & 1.9 & 3.7 & 6.9 & 1.00 & 1.03 & 1.26 & $100 \%$ \\
\hline \multirow{3}{*}{$\begin{array}{l}\text { Kodak } \\
\text { DC290 }\end{array}$} & Raw & 2.2 & 7.2 & 13.8 & 1.00 & 1.03 & 1.19 & $100 \%$ & \multirow{3}{*}{ Sigma SD9 } & Raw & 3.8 & 8.0 & 14.1 & 1.00 & 1.03 & 1.23 & $100 \%$ \\
\hline & Q90 & 1.1 & 2.7 & 5.4 & 1.00 & 1.04 & 1.24 & $93.3 \%$ & & Q90 & 1.4 & 3.2 & 6.9 & 1.00 & 1.03 & 1.25 & $95.6 \%$ \\
\hline & Q75 & 1.0 & 1.4 & 2.2 & 1.00 & 1.03 & 1.23 & $48.9 \%$ & & Q75 & 1.0 & 1.5 & 3.7 & 1.00 & 1.04 & 1.24 & $55.6 \%$ \\
\hline
\end{tabular}

Table 1: Minimum, median, and maximum PSR and probability of detection (PDM) for tested image pairs from all cameras. The decision threshold was set so that the probability of false alarms was $P_{F A} \cong 5 \times 10^{-5}$.

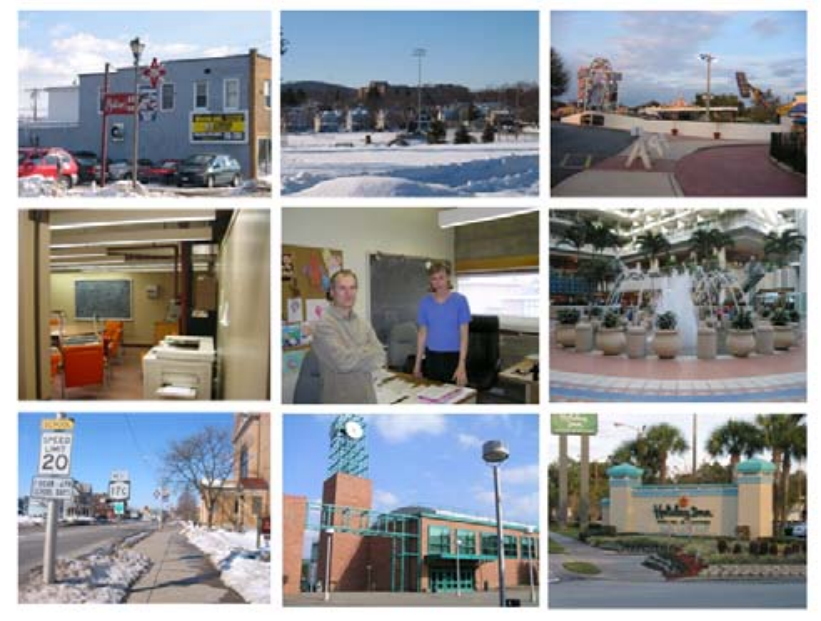

Figure 2: Some sample images used in our tests.

\section{REFERENCES}

[1] Kharrazi, M., Sencar, H.T., and Memon, N.: "Blind Source Camera Identification”, Proc. ICIP’ 04, Singapore, October 24-27, 2004.

[2] Bayram, S., Sencar, H.T., and Memon, N.: "Source camera identification based on CFA interpolation,” ICIP 05, Genoa, Italy, September 2005.

[3] Swaminathan, A., Wu, M., and Liu, K.J.R.: "Non-intrusive Forensic Analysis of Visual Sensors Using Output Images,” IEEE Int. Conf. on Acoustics, Speech, and Signal Processing (ICASSP'06), May 2006.
[4] Geradts, Z., Bijhold, J., Kieft, M., Kurosawa, K., Kuroki, K., and Saitoh, N.: "Methods for Identification of Images Acquired with Digital Cameras", Proc. of SPIE, Enabling Technologies for Law Enforcement and Security, vol. 4232, pp. 505-512, February 2001.

[5] Lukáš, J. Fridrich, J., and Goljan, M.: "Digital Camera Identification from Sensor Pattern Noise,” IEEE Transactions on Information Security and Forensics, vol. 1(2), pp. 205214, June 2006.

[6] Chen, M., Fridrich, J., and Goljan, M.: "Digital Imaging Sensor Identification (Further Study),” Proc. SPIE Electronic Imaging, Security, Steganography, and Watermarking of Multimedia Contents IX, vol. 6505, San Jose, California, January 28 - February 1, pp. 0P-0Q, 2007.

[7] Cox, I., Miller, M.L., and Bloom, J.A.: Digital Watermarking, Morgan Kaufmann, San Francisco, 2001.

[8] Healey, G. and Kondepudy, R.: "Radiometric CCD Camera Calibration and Noise Estimation," IEEE Transactions on Pattern Analysis and Machine Intelligence, vol. 16(3), pp. 267-276, March, 1994.

[9] Mihcak M.K., Kozintsev, I., and Ramchandran, K.: "Spatially adaptive statistical modeling of wavelet image coefficients and its application to denoising," in Proc. IEEE Int. Conf. Acoustics, Speech, and Signal Processing, Phoenix, Arizona, vol. 6, pp. 3253-3256, March 1999.

[10] Holt, C.R.: “Two-Channel Detectors for Arbitrary Linear Channel Distortion,” IEEE Trans. on Acoustics, Speech, and Sig. Proc., vol. ASSP-35(3), pp. 267-273, March 1987.

[11] Vijaya Kuma, B.V.K. and Hassebrook, L.: "Performance Measures for Correlation Filters,” Appl. Opt. 29, 2997-3006, (1990) 